\title{
Unfolding of neutron spectra with an experimentally determined diamond detector response function
}

\author{
Andreas Zimbal', Marcel Reginatto, Miroslav Zbořil \\ Physikalisch-Technische Bundesanstalt, \\ D-38116 Braunschweig, Germany \\ E-mails: Andreas.Zimbaleptb.de, Marcel.Reginattolptb.de, \\ Miroslav.Zborileptb.de
}

\section{Francis Gagnon-Moisan}

Paul Scherrer Institut,

CH-5232 Villigen, Switzerland

E-mail: francis.gagnonepsi.ch

Radiation detectors made from artificial chemical vapor deposition (CVD) single crystal diamond have shown great potential for neutron spectrometry. The detectors are small, typically about $(5 \times 5 \times 0.5) \mathrm{mm}^{3}$, they are not very sensitive to gamma radiation, and they have good radiation hardness properties. They are, therefore, very promising candidates for applications where high resolution neutron spectrometry in very high neutron fluxes is required, such as in fusion research.

A measurement with a single crystal CVD diamond detector results in a pulse height spectrum which contains information about the energy spectrum of the incident neutrons. Unfolding methods can be used to extract this information, but this requires a response matrix. Current particle transport codes, while able to provide important information, are of limited use because they cannot simulate neutron responses of CVD diamond detectors that are of high enough quality to be used for unfolding. Consequently, we have determined the neutron response matrix from measurements.

The response matrix covers the energy range $10.0 \mathrm{MeV}<E_{\mathrm{n}}<16.0 \mathrm{MeV}$. It is based on six measurements of monoenergetic neutron beams produced at the Physikalisch-Technische Bundesanstalt (PTB) ion accelerator facility (PIAF). A Bayesian approach that incorporates signal-background separation techniques and thin plate spline radial basis function interpolation was used to get the full response matrix from this rather limited amount of data. To test the quality of the response matrix, we have done unfoldings of additional measurements with monoenergetic neutron spectra. These were also made at PIAF. The unfolded spectra are in good agreement with numerical simulations of these spectra.

First EPs Conference on Plasma Diagnostics - $1^{\text {st }}$ ECPD

14-17 April 2015,

Villa Mondragone, Frascati (Rome) Italy

${ }^{1}$ Speaker 


\section{Introduction}

Neutrons interact with a diamond detector through different nuclear reactions on carbon. The charged particles that result from these reactions are stopped and deposit their energy in the diamond crystal. The deposited energy $E_{\text {dep }}$ of each event is used to create a pulse height spectrum (PHS), which is a histogram of these events. The response function of the detector is the complete set of the PHS produced by monoenergetic neutrons for a given energy range, normalized to unit fluence.

In a previous paper [1], we presented a Bayesian method to estimate the neutron response function of the detector for a continuous range of neutron energies $\left(10.0 \mathrm{MeV} \leq E_{\mathrm{n}} \leq 16.0 \mathrm{MeV}\right)$ based on a few measurements with quasi-monoenergetic neutrons [2]. This approach was developed because a complete set of measurements is not available and the alternative approach of using responses based on Monte Carlo calculations is not feasible. Current particle transport codes, while able to provide important information, cannot simulate neutron responses of diamond detectors that are of high enough quality. This is because the physics of the detector is not completely understood and the description of the reactions that take place when neutrons interact with carbon needs to be improved.

One of the motivations for doing this work concerns the analysis of measurements in unknown neutron fields. Measurements with a diamond detector result in pulse height spectra which contain information about the neutrons incident on the detector. Knowledge of the neutron response function for an appropriate range of neutron energies makes it possible to use unfolding procedures to obtain information about the energy spectrum of the neutrons. In this paper, we report on unfoldings of measurements that were carried out at the PhysikalischTechnische Bundesanstalt (PTB) ion accelerator facility (PIAF). These unfolding provide a test of the quality of the response matrix and of the soundness of the unfolding procedures used to analyze the data. The unfolded spectra are in good agreement with Monte Carlo simulations of these spectra carried out with the TARGET code [3].

The paper is organized as follows. In the next section we discuss the diamond detector measurements carried out at PIAF using monoenergetic neutron beams. Then we give a brief summary of the method that was used to determine the response function. After that, we discuss the unfolding of the diamond detector data and present our results. We end with some concluding remarks.

\section{Experimental setup}

\subsection{The PTB ion accelerator facility PIAF}

The measurements used to determine the response function and the ones used for the unfolding were done during two measurement campaigns with different experimental setups.

For the measurements used for the response function determination, neutrons were produced using the PIAF cyclotron and the reaction $\mathrm{D}(\mathrm{d}, \mathrm{n})^{3} \mathrm{He}$. The deuteron beam impinged on a $\mathrm{D}_{2}$-filled gas target. Above a deuteron energy of $8.9 \mathrm{MeV}$, the deuteron breakup leads to a low- 
energy continuum in addition to the monoenergetic neutrons. This continuum was separated from the monoenergetic neutrons using time of flight methods [2].

For the data used for the unfolding, neutrons were produced via the $\mathrm{T}(\mathrm{d}, \mathrm{n})^{4} \mathrm{He}$ reaction. The deuteron beam $\left(E_{\mathrm{d}}=215 \mathrm{keV}, I \sim 10 \mu \mathrm{A}\right)$ from the Van de Graaff accelerator impinged on a rotating solid $\mathrm{Ti}(\mathrm{T})$ target. Monoenergetic neutrons of $14.0 \mathrm{MeV}$ and $14.8 \mathrm{MeV}$ were realized by choosing neutron emission angles of $98^{\circ}$ and $0^{\circ}$ with respect to the deuteron beam, respectively. The distance between the target and the detector was $25 \mathrm{~cm}$ [4].

\subsection{Diamond detectors}

The measurements were carried out using two different detectors from Diamond Detectors Ltd. The nominal dimensions of the detectors are $4.6 \times 4.6 \times 0.5 \mathrm{~mm}^{3}$, but their electrodes differ in size and geometry. The detector for the measurements that were used to determine the response function has circular electrodes while the detector for the measurements that were used for the unfolding has square electrodes, but this does not affect strongly the response. For both sets of measurements, standard analog electronics was used (see Refs. [2] and [4] for details).

\section{Experimentally determined response function}

The data that we used for the experimentally determined response function is shown in Fig. 1. The mean neutron energies of the six beams are $E_{\mathrm{n}}=10.08,11.43,12.61,13.71,14.96$, and 16.01 MeV. Further details are available in Ref. [2].

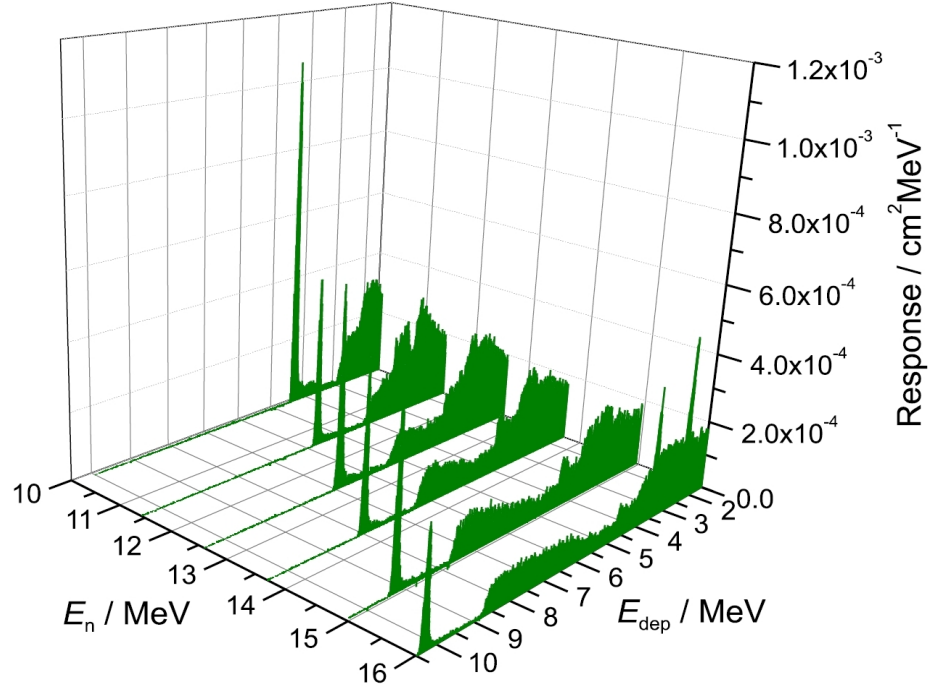

Figure 1: Measurements of the response of the diamond detector to monoenergetic neutrons [1].

To determine the response function from the data, we used Bayesian methods [1]. To carry out the analysis, we modeled each of the measured PHS in terms of two additive components, a smooth background and a signal with fine structure (e.g., peaks). Our approach uses Bayesian signal-background separation techniques to carry out the joint estimate of the signal and background. The separation into signal and background leads to a great simplification. The 
interpolation of the background is relatively straightforward because it is a smooth function, and it can be carried out using thin plate spline (TPS) radial basis functions (RBF) interpolation. The interpolation of the fine structure that is assigned to the signal requires more care, and for this we combine information from measurements with cross section data and information from simulations [5]. The overall normalization of the response function has an uncertainty that we estimate is of the order of $10 \%$, which is due to the fact that an absolute normalization for each of the measured PHS has not been completed yet. For more details of the analysis, see Ref. [1]. The response function determined in this way is shown in Fig. 2.

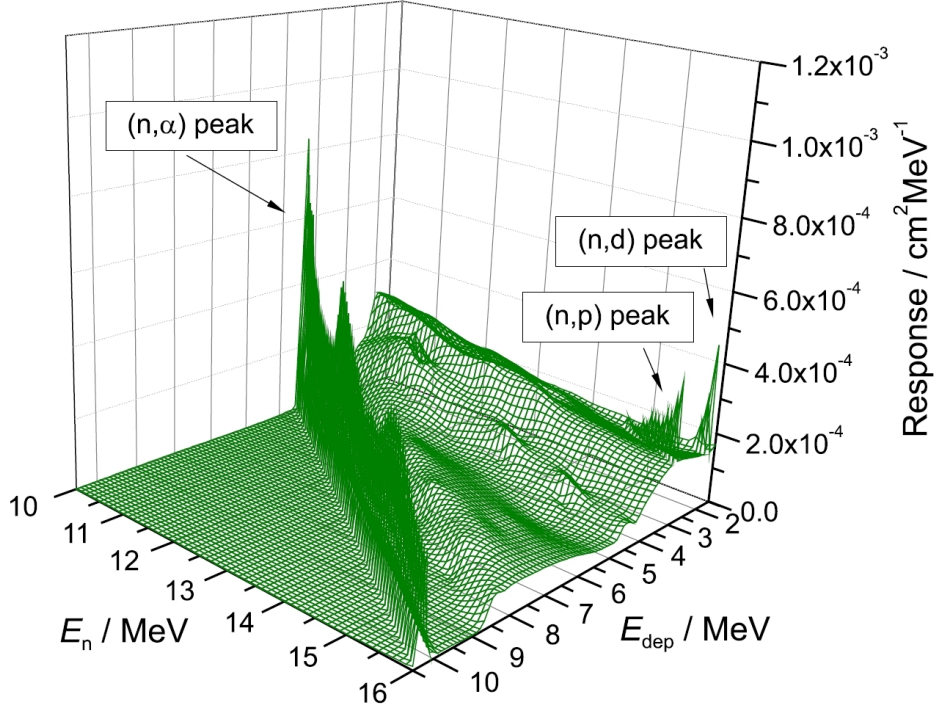

Figure 2: Response function of the diamond detector derived from the measurements shown in Fig. 1 using a Bayesian approach [1].

\section{Unfolding of the diamond detector data}

A measurement carried out with a diamond detector provides an indirect measurement of the neutron energy spectrum $\varphi_{E}(E)$ and an unfolding procedure is needed to determine $\varphi_{E}(E)$. For practical applications, a discrete energy binning is introduced and $\varphi_{E}(E)$ is replaced by the fluence vector $\varphi_{l}$. The PHS is related to the fluence vector by the linear equation [6]

$$
N_{k}+e_{k}=\sum_{l} R_{k l} \varphi_{l}
$$

where $N_{k}$ is the number of counts in channel $k$ of the PHS, $R_{k l}$ is the response matrix and $e_{k}$ is a term which accounts for effects that are not described by the model of the measurement; e.g., statistical fluctuations in the number of counts, discrepancies between $N_{k}$ and $\int R_{k}(E) \varphi_{E}(E) d E$ due to deviations of $R_{k}(E)$ from the true value of the response, etc. An unfolding method is a mathematical procedure that selects a fluence vector $\varphi_{l}$ which solves Eq. (1) [6,7]. We did the unfoldings with the code GRAVEL which is part of the UMG 
package [8]. GRAVEL is an iterative code which starts from a default spectrum (i.e., an initial estimate of the spectrum) and produces a solution spectrum $\varphi_{l}$. For the unfoldings, we used a constant default spectrum, an energy range for the PHS of $2.0 \mathrm{MeV}$ to $10.0 \mathrm{MeV}$, and an energy range for the neutron energy of $10.0 \mathrm{MeV}$ to $15.8 \mathrm{MeV}$.

Figs. 3 and 4 show the results of the unfoldings for the $14.0 \mathrm{MeV}$ and $14.8 \mathrm{MeV}$ measurements, together with the neutron energy spectrum simulations for these experimental conditions which were calculated with the TARGET code [3]. Due to the uncertainty in the absolute normalization of the response function and the use of a different diamond detector for these measurements, we have normalized the peak areas $\left(13.5 \mathrm{MeV}<E_{\mathrm{n}}<15.5 \mathrm{MeV}\right)$ of the unfolded and simulated spectra to unit fluence. The inset shows a comparison of the measured PHS and the PHS obtained by folding the solution spectrum $\varphi_{l}$ with the response function.

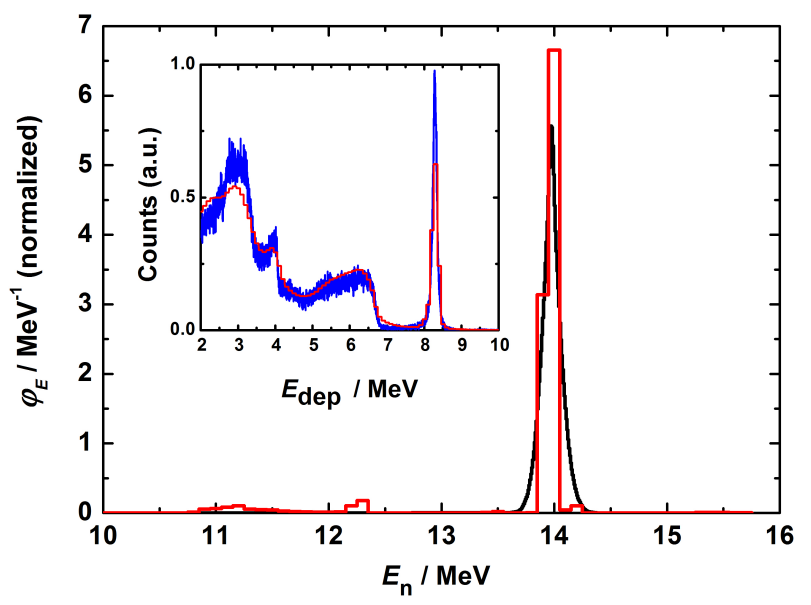

Figure 3: Unfolded spectrum (red) and TARGET code simulation (black) for monoenergetic neutrons with $E_{\mathrm{n}}=14.0 \mathrm{MeV}$. The inset shows the measured PHS (blue) and the PHS that results from folding the response function with the unfolded spectrum (red).

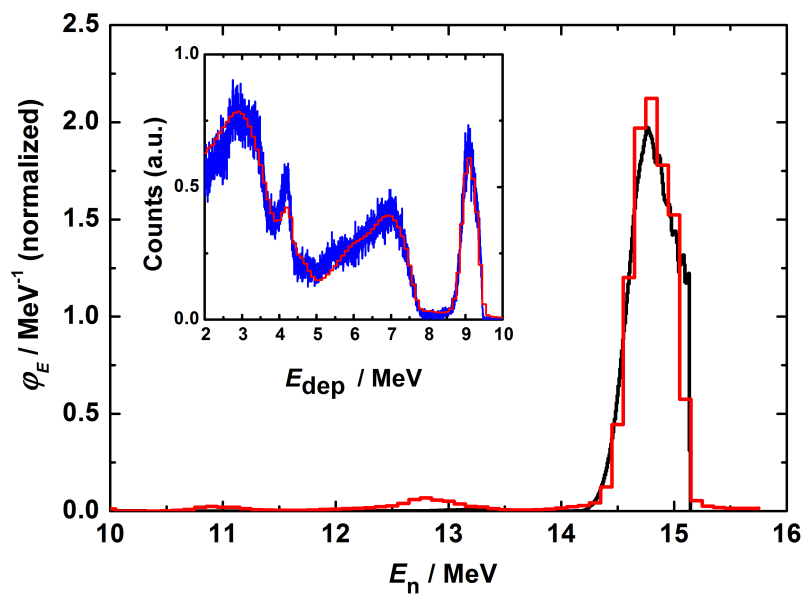

Figure 4: Unfolded spectrum (red) and TARGET code simulation (black) for monoenergetic neutrons with $E_{\mathrm{n}}=14.8 \mathrm{MeV}$. The inset shows the measured PHS (blue) and the PHS that results from folding the response function with the unfolded spectrum (red). 


\section{Discussion and concluding remarks}

There is good agreement between the unfolded spectra and the simulations done with the TARGET code, as can be seen from Figs. 3 and 4 . This provides a validation of the experimentally determined response matrix and of the method used for the unfolding. Notice that the results achieved with the unfolding using the experimentally determined response matrix are such that the shape of the peaks are in good agreement with the simulations.

The use of unfolding methods and experimentally determined response functions allows neutron spectrometry with diamond detectors in unknown fields. We have shown two examples of unfolding of measurements of monoenergetic neutrons which are challenging due to the relatively small width of the peaks. It is clear, however, that the same procedure can be used to unfold measurements of broad distributions or of peaks with long tails. This is of interest for neutron diagnostics in fusion facilities, where the shape and the width of the DT fusion $14 \mathrm{MeV}$ peak contain important information about the plasma conditions.

We plan to improve the Bayesian method of Ref. [1] that was used to determine the response function and to extend the response function to cover a wider energy range. Furthermore, we continue to improve the numerical simulations of the diamond detector responses [5]. Additional measurements and unfoldings will be carried out to further test and improve both the response function and the unfolding procedures.

\section{References}

[1] M. Reginatto, F. Gagnon-Moisan, J. Guerrero Araque, R. Nolte, M. Zbořil and A. Zimbal, A Bayesian method to estimate the neutron response matrix of a single crystal CVD diamond detector, AIP Conference Proceedings 1641, 321 (2015).

[2] F. Gagnon-Moisan, A. Zimbal, R. Nolte, M. Reginatto, and H. Schuhmacher, Characterization of single crystal chemical vapor deposition diamond detectors for neutron spectrometry, Rev. Sci. Instrum. 83, 10D906 (2012).

[3] D. Schlegel, TARGET User's Manual, Laboratory Report PTB-6.42-05-2 (2005).

[4] M. Zbořil and A. Zimbal, Response function stability of single crystal diamond detectors to $14 \mathrm{MeV}$ neutrons, Rev. Sci. Instrum. 85, 11D839 (2014).

[5] M. Zbořil, J.E. Guerrero Araque, R. Nolte, and A. Zimbal, Simulation of the neutron response functions of diamond detectors with the NRESP code, POS (ECPD2015) 098

[6] M. Reginatto, Overview of spectral unfolding techniques and uncertainty estimation, Rad. Meas. 45, 1323 (2010).

[7] M. Reginatto and A. Zimbal, Bayesian and maximum entropy methods for fusion diagnostic measurements with compact neutron spectrometers, Rev. Sci. Instrum. 79, 023505 (2008).

[8] M. Reginatto, B. Wiegel, and A. Zimbal, UMG 3.3 - Unfolding with MAXED and GRAVEL, available from the NEA Data Bank at http://www.oecd-nea.org/tools/abstract/detail/nea-1665 and from RSICC at https://rsicc.ornl.gov/codes/psr/psr5/psr-529.html. 